\title{
Application research of PLC in the CNC system Yun-Kuan JIANG ${ }^{1}$ \\ Department of mechanical engineering , Liaoning Jidian Polytechnic, Dandong , china \\ E-mail:176949746@qq.com
}

Keywords: PLC control; Digital control; CNC system; Safety interlock

\begin{abstract}
PLC is a kind of special order to the industrial control microcomputer system.In order to adapt to the requirement of sequence control,Out the microcomputer some Numbers of PLC function, and strengthen the function of logic control,Is a kind of between relay control and computer control automatic control device;PLC is designed for use in harsh industrial environments, so has a strong anti-interference ability.In addition to the input/output part adopts photoelectric isolation measures, the power supply, arithmetic unit, controller, storage and so on also set up many kinds of protection and shielding.PLC is not relay the mechanical contact, therefore, there is no contact of poor contact, fusion welding, wear and damage of coil, etc.
\end{abstract}

\section{Introduction}

Modern CNC machine tools PLC instead of relay control to complete the logical control, numerical control machine tool structure is more compact, more feature-rich, responsiveness and reliability greatly improved[1]. Programmable controller is the especially for the application in industrial environments and the design of digital electronic systems of arithmetic operations, it uses a programmable program memory, performs a logical calculation in its internal storage, order control, timer, counting and arithmetic operations such as operation instruction, through digital, analog inputs and outputs to control various types of mechanical equipment and production processes. Combined machine tool is designed for a particular job, specific processing and design of a highly efficient automated processing equipment, such equipment most of more than one tool at work, and has the automatic circulation function.

Usually consists of modular machine tool general part and special part combination for standard, power part with motor or hydraulic systems drive, automatic circulation control by electrical systems, is typical of the mechanical and electrical or mechanical-electrical-hydraulic integration of automatic processing equipment.

\section{THE WORKING PRINCIPLE OF PLC}

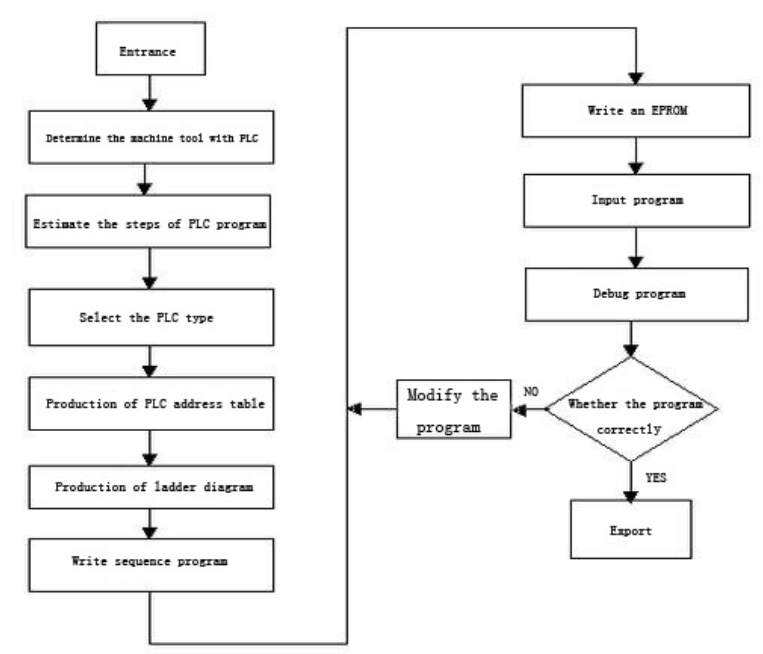

Fig. 1 The design of the machine tool control program flow chart 
Relay control device with the method of logical run in parallel, namely if the relay coil electricity or without electricity, all the relay contacts (including their normally open or normally closed contacts) on the relay control circuit which position will immediately action at the same time.PLC of the CPU, the use of the sequential logic scan user program operation mode, if an output coil or logical coil is on or off, all the coil contact (including their normally open or normally closed contacts) no immediate action, must wait for scan will move to the contact.[2]The desing of the machine tool control program flow chart is shown in figure 1 above.

So-called from PLC I/O response time refers to an input signal changes to the system about the time needed for the change of the output signal.I/O response time the shortest and the longest I/O response time as shown figure 2 below.

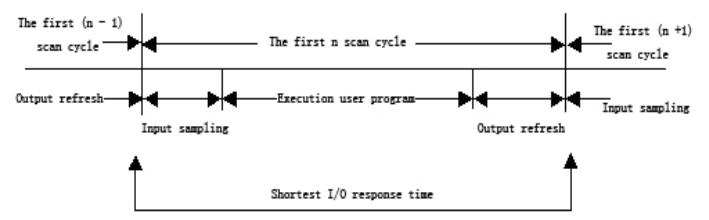

Fig.2 The shortest I/O response time

Above is the general working principle of PLC, but in the modern emergence of more advanced PLC input image refresh cycle, the program execution cycle and output image refresh cycle have been able to work independently, to improve the execution efficiency of PLC.In practical engineering application, the programmer should know the working principle of the above to write a good quality, high efficiency of processes.

\section{The relationship between PLC and NC}

Automatic control of PLC for general equipment, called the programmable controller.PLC used in the periphery of the CNC machine auxiliary electrical control, called the programmable controller of the machine.Therefore, in a lot of numerical control system is the PMC (programmable machine tool controller).[3]Numerical control system has two parts, one is NC, the other is PLC, both in the scope of the role of the NC machine tool is not the same.It can be divided into the scope of NC and PLC:

1 Realize the cutting tool relative to the workpiece axis geometry motion law of digital control. This task is performed by NC;

2 Machine auxiliary equipment control is accomplished by PLC.

It is in the process of nc machine tool operation, according to the CNC internal as well as the control switch of the machine tool, detecting element, the state of running parts, in accordance with the procedures set by the control logic of such as dao library movement, changing mechanism, the operation of the cooling liquid, etc.

\section{The application of PLC in the numerical control machine tool}

A.Safety interlock

If there are A, B two process, for security purposes, when action will limit after B action, said A to B with safety interlock;If B has A safety interlock, said of A and B ask for two-way safety interlock, hereinafter referred to as safety interlock between A and B. PLC and RLC except the complete must function also requires that in the case of abnormal through safety interlock to the operator and equipment for emergency protection, and then through the alarm to notify the operator work, therefore, must understand in detail in the design of equipment working process and principle, link analysis easy to accident, so as to achieve in the program and the electrical design.Also consider the PLC program may not be perfect, or software failures occur, some important protection function must be implemented by hardware as RLC.We usually call the design of the RLC electric design, the design of PLC software design.Here are in general need to pay attention to in the design of software and hardware of safety interlock function.[4] 


\section{B.scram}

When there is a tight when the situation is urgent, you can press the stop button to avoid the further failure and accident, therefore, abrupt stop button must be able to stop the moving parts, such as feed shaft, spindle, tool post, commonly used method is, through the urgent stop button to close all the power source, only keep control power: also can use to stop button to close all the moving parts and drive can make the signal to implement, but if the drive is out of control this kind of method is invalid.Stop safety interlock and must be implemented in the electrical design.

\section{C.limit}

When the feed shaft or other moving parts with position requirements beyond the design of the trip, through limit signal for protection.Limit signals on the one hand, need to ban on the movement of the moving parts in a limit moment; , on the other hand, also notice the direction of the NC limit, such as with overtravel lift and otherwise out of limit state, won't because of wrong operation and further expand over travel, which allows only moving parts move on and limit in the opposite direction to the limit of safety interlock in electrical design and software design two aspects of the implementation.

D.Feed drive

NC machine tools in the automatic processing zhongtong to exercise regularly is driven by more than one feed device at the same time, therefore, when a feed drive appear alarm, must stop automatically processing status. The feed drive safety interlock is realized in software.

E.Spindle unit

Nc machine tool spindle rotary motion to drive the cutting tool or workpiece cutting movement.(1) when the spindle alarm automatic processing must be forbidden, in order to prevent damage to the cutting tool or spindle [5]:(2) when the main shaft movement, must ban knives loose/tight and the process of automatic tool change etc need spindle static;Have multiple block (3) if the spindle, the spindle shift not success must be forbidden continuous movement and automatic processing of machine tool spindle.The above (1) and (3) general implementation in software design;And (2) a realization in the electrical design

F.Three-phase asynchronous motor commutation

Three-phase asynchronous motor through out in any two phase power, to change the direction of rotation of the motor, as shown in figure l.If control the forward and inversion of two ac contactor closed at the same time, the two meet in the three-phase power supply to short circuit, so the two ac contactor control must be performed to the safety interlock as shown in figure 3 .
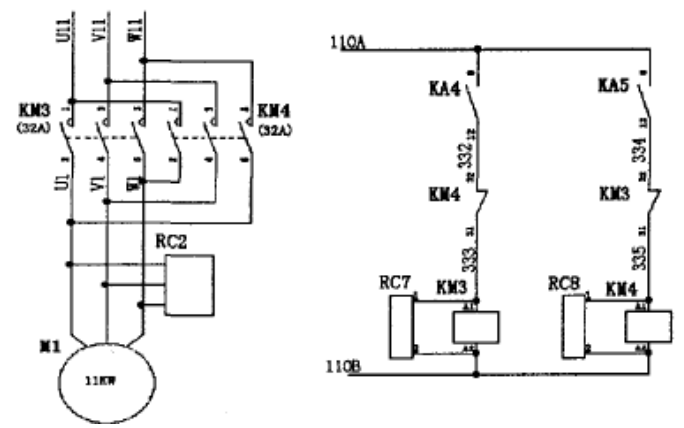

Fig.3 Two-way safety interlock

And the release of ac contactor has certain hysteresis, if two ac contactor control loops have electricity at the same time, there will be a transient on instantaneous short circuit makes the process of the power supply at the same time, so in the software design is also had to design the safety of forward and reverse interlocking.

G.CNC machine tool design and debugging of PLC system

Now each function of nc machine tools, especially the general nc machine tools, such as the spindle control transposition, machining center, lathe tool slide knife library, lubrication and cooling of the start/stop has been standardized, all kinds of CNC system are built-in or provide the PLC program can satisfy the work.Using independent model of PLC, general manufacturers also provide standard PLC program that could satisfy the requirement of general nc machine 
tools.Design PLC program, therefore, the most important method is detailed understanding and reference for the system to provide the standard PLC program.The PLC program design are the key problems in the application.PLC program design in accordance with the requirements of the equipment is the basic train of thought of design input and output signal of the logic relationship, to get the desired output signal when the input some signal, so as to realize the expected work process.So simple and common method is a process for the target, analysis of each process starting conditions and constraints, the PLC program of the process according to these conditions, finished all process of PLC program to complete the entire PLC program.One of the process can involve an output interface, such as cooling motor start/stop;Can also involve multiple output to 511, such as the process of machining center tool change.This method is easier to achieve PLC program of modularization, easy to the process of independent debugging.

\section{Conclusion}

Starting from the nc machine tools was born in 1952 in our country from an agricultural country for the development of a machine tool production and application of power, but the numerical control technology application level is not high, seriously restricts the improvement of China's manufacturing industry.Relevant international development plan for the development of numerical control technology in our country put forward the serious challenge, at the same time also brought opportunities.Only to choose the appropriate PLC to achieve the desired effect.Yonghong FBS series NC positioning function of PLC in machine tool numerical control system of the machine after a long run shows that the whole system design is reasonable, high control accuracy, reliable operation, improves the automation level of production, reduce the labor intensity of the operators.With PLC control, increase the anti-interference ability of the electric parts, improve the reliability of the machine tool and thus increased the flexible equipment, improve the efficiency of the use of the equipment.

\section{Acknowledgement}

This thesis project support: The numerical control vocational skill training and the numerical control specialized core curriculum teaching research and practice of butt fusion Item number: JYLX2013003

\section{References}

[1] YuHanQi, Guo Jian principle and application of programmable controller in Beijing: China powerpress, 2004

[2] section Su Zhen design factors to enhance the reliability of PLC control system of electric drive, 2003

[3] Zhang Xiaoqi mechanical and electrical equipment control based in Beijing, China's National $\begin{array}{lll}\text { People's Congress } & \text { press, } & 2000\end{array}$ [4] Wang Zhaoyi small programmable controller practical technology Beijing: mechanical industrypress, 1997

[5] mitsubishi plait 1 fx series PLC instructions and programming manual, 1998 